\title{
Chatten als Kommunikationsspiel
}

\author{
Susanne Wolf / Helena Bilandzic
}

Ausgehend vom Rabmenkonzept Goffmans wird ein Spielmodell des Chattens entwickelt, das eine weiter gehende Erklärung der Chatnutzung bietet. Mit Hilfe des Modells lässt sich zeigen, warum Anonymität und Unverbindlichkeit als grundlegende Charakteristika der Chatkommunikation nicht zu einer völligen Missachtung von Gesprächs- und Umgangskonventionen fübren. Das Spielmodell basiert auf den Grundelementen konventioneller Spiele und wurde in einer empirischen Studie mit einer Kombination von Beobachtung und Lautem Denken konkretisiert. Als Spielhandlungen werden die einzelnen Kommunikate der Chatter betrachtet. Die Darstellung der eigenen Identität zu steuern und die des anderen zu enttarnen, kann als spielimmanentes Ziel begriffen werden. Dabei geht es nicht um das bloße Entlarven des Gesprächspartners, vielmehr ist ein origineller und geistreicher Weg dorthin - ̈̈ber die Spielhandlungen entscheidend. Dementsprechend entwickeln die Chatter Spielstrategien, um ibre Kommunikationsgeschicklichkeit zu steigern: Aktivität, Schnelligkeit und Originalität machen einen Spieler zum gefragten Gesprächspartner. Beherrscht man diese Strategien nicht oder missachtet man die grundlegenden Umgangsformen, wird man im Chat ignoriert und aus dem Spiel ausgeschlossen - mit der Konsequenz, dass auch das Spielvergnügen beendet ist.

Keywords: Chat, Spiel, Rahmenanalyse, lautes Denken

\section{Einführung}

Pessimisten warnten Anfang der 90er Jahre vor negativen Auswirkungen der ChatKommunikation: Anonyme Netzbekanntschaften würden reale Beziehungen ersetzen und zu sozialer Isolation führen (z. B. Mettler-Meibom, 1990). Nachdem die erste Aufregung über die neue Kommunikationsform Chat abgeklungen ist, sowohl bei Kritikern als auch Nutzern, kann man sagen, dass sich die Befürchtungen nicht bewahrheitet haben. Obwohl 30\% aller Onliner den Chat nutzen und dies unter anderem auch deshalb tun, um Kontakte zu knüpfen, sind es relativ wenige, die ihre realen Bekanntschaften wegen Cyberspace-Beziehungen vernachlässigen (Döring, 1996: 300). Neben den Kontaktmotiven gibt es eine weitere wichtige Gruppe von Motiven, warum sich Menschen der Chatkommunikation zuwenden; das sind unspezifische Motive wie Unterhaltung, Zeitvertreib, Ablenkung. Um diese unspezifischen Motive zu konkretisieren und eine weiter gehende Erklärung der Chatnutzung zu bieten, schlagen wir in diesem Beitrag vor, den Chat als Kommunikationsspiel zu betrachten. Nach einer theoretischen Analyse der Kommunikationssituation im Chat und ihrer Konsequenzen als Spielvoraussetzungen wird der Spielaspekt empirisch untersucht.

\section{Theoretische Analyse des Chat}

Das Gespräch im Chat trägt die typischen Merkmale eines informellen Alltagsgesprächs (vgl. Gesprächstyp-Dimensionen nach Henne und Rehbock, 1995: 32 f.): Es ist ein natürliches Gespräch, das sich erst im Verlauf konstituiert und nicht vorbereitet ist; die Handlungsdimensionen sind offen, sowohl diskursive (kontroverse Diskussion von Normen 
oder Themen), direktive (Handlungs- und Hinweischarakter, etwa Ratsuche, Ratgeben) als auch narrative (ohne bestimmtes inhaltliches Ziel zur Herstellung eines sprachlichen Kontaktes) Gespräche scheinen im Chat möglich. Diese geringe Determiniertheit macht das Alltags- sowie das Chatgespräch gewissermaßen selbstzweckhaft: Nicht der Gesprächsinhalt ist (in der Regel) wichtig, sondern dass das Gespräch läuft dem „Gesprächsfluss wird nahezu jeder Gesprächsgegenstand geopfert“ (Keppler, 1994: $88 \mathrm{f}$.$) .$

Der Chat stellt eine Fernkommunikation dar, die zwar zeitlich simultan, aber räumlich fern abläuft. Dies ist ebenso beim Telefonieren der Fall, jedoch schrumpft die räumliche Nähe beim Chat so weit, dass jegliche Körperlichkeit, etwa das Sehen der Person, aber auch das Hören ihrer Stimme, wegfällt. Das Fehlen des physisch-interpersonellen Kontexts, das bereits oft als wichtigstes Charakteristikum des Chat wie der Netzkommunikation überhaupt genannt worden ist (z. B. Gallery 2000: 71), setzt die Bedingungen für die Kommunikation.

Anonymität. Durch das Wegfallen des physisch-interpersonellen Kontexts fehlen Informationen wie Name, Status, Alter, Geschlecht und Aussehen und machen die visuelle oder auditive Identifikation der Real-Life-Identität einer Person unmöglich - die Kommunikationspartner können anonym bleiben. Allerdings herrscht keine völlige Namenlosigkeit im Chat, da Nicknames zur Kennzeichnung der Chat-Persönlichkeit dienen. $\mathrm{Ob}$ die Angabe dieses fiktiven Namens jedoch ausreicht, um die Anonymität im Chat aufzubeben, ist stark zu bezweifeln (Gallery 2000: 72). Gerade Kategorien wie Geschlecht und Alter spielen eine entscheidende Rolle in der sozialen Interaktion, nicht zu vergessen auch Hautfarbe, Größe, Attraktivität etc. Solche Merkmale fungieren als Schlüssel, mit denen eine ganze Reihe von Erwartungen verbunden sind.

Unverbindlichkeit. Des Weiteren fehlt der Kommunikation im Chat die Verbindlichkeit der direkten Kommunikation. Mediale Kontakte entbehren gegenseitiger Verpflichtungen und Zusagen, die bei realen Begegnungen eingegangen werden (Höflich, 1999: 147; Turkle, 1999: 286). Direkte und unverblümte, auch verletzende Äußerungen (flaming) können ohne Konsequenzen für die Real-Life-Existenz erfolgen (Kiesler/Siegel/McGuire, 1984: 1129). Interessanter Weise herrscht aber trotzdem ein großes Maß an Selbstkontrolle vor; ungezügelte Äußerungen halten sich in Grenzen (Klemm/Graner, 2000: 165).

Nicht nur die Verantwortung für die Folgen einer Äußerung ist schwerlich einzufordern, auch grundlegende Gesprächskonventionen, wie etwa, dass der Gesprächspartner die volle Aufmerksamkeit erhält, oder dass nebenbei nicht geflüstert wird, können unbemerkt gebrochen werden. Ebenso kann die Verbindlichkeit zu einer Kontinuität des Gesprächs verletzt werden: „Relativ mühelos kann man sich in ein Gespräch einschalten und wieder ausklinken“ und, so Scherer und Wirth weiter, „man hinterlässt zwar Spuren, kann aber nicht aufgespürt werden“ (Scherer/Wirth, 2002: 341).

Handlungen im Chat. Diese besonderen Umstände der Chat-Kommunikation wirken auf die Kommunikation selbst zurück. Diese Anpassung an den Kontext kann mit dem Rahmenkonzept von Goffman theoretisch formuliert werden (Goffman, 1977; Esser, 1999; vgl. auch Höflich, 1998, 1999; Höflich/Gebhardt 2001; Döring, 1997a: 303): Menschen finden sich in ihrem Alltag zurecht, indem sie sich in jeder Situation fragen, was gerade vor sich geht. Dadurch definieren sie die aktuelle Situation, oder anders ausgedrückt, sie geben ihr einen Rahmen, der wiederum ihre folgenden Handlungen wesentlich bestimmt. Mit dem Begriff Rahmen (Frame) sind Deutungs- und Interpretationsmuster gemeint, über die Konsens bei den Akteuren herrscht, und die eine soziale Situation aus der Sicht der Akteure strukturieren und Verhaltenserwartungen schaffen 
(Döring, 1997a: 303; Hettlage, 1991: 95-154). Ein klarer Rahmen liegt dann vor, wenn der Konsens stark ist, die Beteiligten die Situation also ähnlich einschätzen. Beim Chat wird der Rahmen vor allem durch das Fehlen des physisch-interpersonellen Kontexts sowie der daraus resultierenden Anonymität und Unverbindlichkeit geprägt.

Im Gegensatz dazu ist Rahmung (Framing) die Situationsdefinition, wie sie konkrete Akteure in einer konkreten Situation durchführen. Sie kann eine Aktualisierung des Rahmens enthalten, aber auch darüber hinausgehen, vor allem, wenn der Rahmen unklar ist und neue unvertraute Situationen vorliegen (Goffman, 1977: 46 f.; Crook/Taylor, 1980: 245; Willems 1997: 59). In der konkreten Chat-Situation müssen sich die Teilnehmer fortwährend orientieren, wenn sie ihre sprachlichen Handlungen planen (z. B. Wer ist im Chat? Worüber wird geredet? Wie kann ich mich einbringen?).

Der Chat-Rahmen hat drei Konsequenzen für den Umgang der User mit dem Chat und damit für die Rahmung: (1) Die gegenseitige Wahrnehmung der Kommunikationspartner verändert sich. (2) Die Erwartungen an die Kommunikation werden den Bedingungen angepasst. (3) Die eigene Person wird von ihrer Real-Life-Identität befreit.

1. Veränderte Wahrnehmung der Kommunikationspartner. Im Chat werden soziale Hinweisreize durch die Art der Übermittlung ausgeblendet. In der Terminologie der Kanalreduktionstheorie ist mit der Verringerung der Kommunikationskanäle ein Informationsverlust verbunden, der die Wahrnehmung der Kommunikationspartner verändert (Kiesler/Siegel/McGuire, 1984: 1123 ff.; Döring, 1997b: 278 f.). Gerade die im Chat fehlenden Informationen über den Hintergrund (Alter, Aussehen, Bildung, Status, Vermögen) einer Person entscheiden in Alltagssituationen oft, wie wir eine Person einschätzen, ob sie uns sympathisch ist und ob wir mit ihr in Kontakt treten. Im Chat hingegen haben die Teilnehmer ausschließlich die Kommunikate (Threads) zur Verfügung, um die Situation zu definieren und die Personen einzuschätzen - „unfreiwillige“ nonverbale, paraverbale und sonstige Personeninformationen fließen in die Rahmung nicht ein. ${ }^{1}$ Jede neue Äußerung kann das bisherige Bild vom Gesprächspartner vervollständigen oder revidieren.

Zum anderen geht mit der fehlenden Information ein ständiges Hinterfragen der Glaubwürdigkeit einher: „Glaubwürdigkeit ist das Ergebnis eines konkreten und situativen Zuschreibungsprozesses, in dessen Verlauf der Rezipient eine Botschaft, eine Quelle, ein Medium oder auch andere Sachverhalte überprüft und einschätzt“" (Krotz, 1999: 126). In der Face-to-Face-Kommunikation erfolgt diese Zuschreibung und Überprüfung anhand nonverbaler Aspekte wie Gestik und Mimik. Als Indikatoren für Täuschungen werden z. B. Kopf- und Körperbewegungen, Blickkontakte, aber auch Sprechgeschwindigkeit, Pausen und Stottern herangezogen, die im Chat freilich fehlen. Man muss sich wohl damit arrangieren, so Höflich, im Netz jederzeit einer Täuschung ausgeliefert zu sein (Höflich, 1999: 147).

2. Anpassung der Kommunikationserwartungen an den Chat-Rahmen. Wegen ihrer Besonderheiten kann die Chatkommunikation nicht alle Funktionen der interpersonalen Kommunikation übernehmen. Manche kommunikativen Ziele erscheinen durch eine elektronische Vermittlung geradezu gefährdet (Versöhnungsgespräche, Kondolenzbezeugungen). Eine Abwägung, welches Medium für welches Kommunikationsvorhaben geeignet ist, ist notwendig (Döring, 1997b: 279 f.). Es ist daher plausibel, dass

1 Daher stammt auch die Anfälligkeit der Chat-Kommunikation für Missverständnisse (z. B. bei ironischen Bemerkungen ohne stimmliche oder mimische Ironiezeichen). 
Chatter ihre Erwartungen am Chat-Rahmen ausrichten. Eine Substitution persönlicher Kontakte durch Netzkontakte (z. B. Mettler-Meibom, 1990: 65 ff.) erscheint wenig wahrscheinlich, weil an die beiden Kommunikationsarten verschiedene Erwartungen gerichtet und unterschiedliche Gratifikationen gesucht werden, was eher eine Komplementarität nahe legt (vgl. auch die Befragungsergebnisse von Döring, 1996: 300 und Weinreich, 1997: 84 f.). Netzbeziehungen können zwar ins reale Leben übertragen werden, nehmen dann aber durch den Rahmenwechsel einen völlig neuen Charakter an.

3. „Befreiung“von der eigenen Real-Life-Identität. Krotz sieht die „Anonymität als Chance“ (Krotz, 1999: 125), weil sie von den Zwängen der direkten Kommunikation befreit. So werden z. B. soziale Barrieren verringert und ausgewogenere Partizipationschancen ermöglicht. Eine virtuelle Medienidentität kann beliebig angenommen und durchgespielt werden, ${ }^{2}$ wie z. B. der oft diskutierte Fall eines Psychiaters, der sich als eine nach einem Autounfall gelähmte Frau ausgab, zeigt (van Gelder, 1991: 373; Stone, 1995: 70 ff.). Daneben gibt es auch relativ harmlose Täuschungen, wie die selektive Selbstpräsentation (z. B. Verschweigen überschüssiger Pfunde; Walther, 1996: 19) oder die Selbstkorrektur (Hinzufügen einiger Zentimeter Körpergröße). Es kann sich dabei um Veränderungen handeln, die für die jeweilige Kommunikationssituation funktional sind, etwa die Akzeptanz als Chat-Partner erhöhen, oder dem eigenen Selbstbild besser entsprechen (Scherer/Wirth, 2002: 340, mit Hinweis auf Döring, 1999). Medienidentitäten müssen sich schlüssig präsentieren, sie müssen also bestimmte Rollen erlernen und diese konsequent spielen, was bei einer großen Diskrepanz zur Real-Life-Identität auf Dauer sehr anstrengend ist und hohe Konzentration erfordert (Scherer/Wirth, 2002: 341). Wenn hier von „Identität“ die Rede ist, so sind Schlüsselmerkmale gemeint, die in einem Face-to-Face-Kontakt sofort in Erfahrung gebracht werden können (Alter, Geschlecht, Status, Aussehen) und die als Basisinformation über eine Person mit einer ganzen Grammatik von Erwartungen verbunden sind. Diese Schlüsselinformationen zur Identität, die sonst so selbstverständlich sind, fehlen im Chat.

Soziale Regeln im Netz. Die pessimistische Interpretation der drei beschriebenen Konsequenzen lautet, dass der Chat generell Möglichkeiten zur (böswilligen) Täuschung über die eigene Identität und Verletzungen von Standards des sozialen Umgangs eröffnet. Jedoch „entstehen nicht nur dem Alltag analoge Formen sozialer Vergemeinschaftung (etwa: Kontinuität durch den gleichen Nick, ... usw.), sondern die Chatter beginnen sich darüber hinaus auch als Gemeinschaft zu verstehen und aktiv eine soziale Verregelung zu betreiben“ (Schmidt, 2000: 18), etwa in Form der Netiquetten, deren Einhaltung durch die so genannte elektronische Gemeinschaft überwacht wird (Höflich, 1995: 529). Auch umfassende Täuschungen zur eigenen Identität erfolgen nicht in dem Maße, wie es möglich wäre: Scherer und Wirth (2002) finden in einer Befragung von Chattern heraus, dass die Mehrheit sich im Chat eher authentisch präsentiert. Obwohl mehr als zwei Drittel der Befragten in einer anderen Frage zugeben, beim Aussehen, Alter, Geschlecht oder bei ihren Ansichten etwas zu schummeln, kommen die Autoren zu dem Schluss, dass diese „Schummeleien“ nie so weit gehen, dass sich die Chatter nicht mehr als eigene authentische Persönlichkeit erleben und vielmehr „beziehungs- und

2 Manche Autoren sprechen von den Usern nicht mehr als Personen, sondern als Medienidentitäten oder Chiffrenexistenzen, deren Gebrauch zu einem partiellen Realitäts- und Identitätsverlust führen kann (Klemm/Graner 2000: 156; Höflich, 1998: 149). 
kommunikationstaktische Gründe“ (Scherer/Wirth, 2002: 354) haben als die der Täuschung des anderen über die eigene Identität.

Warum werden die potenziell möglichen Grenzen nicht ausgereizt? Neben der Tatsache, dass die dauerhafte Annahme einer anderen Identität z. B. schwierig und anstrengend ist, sind zwei weitere Erklärungen denkbar:

1. Nach längerer Bekanntschaft werden die Regeln aus der Face-to-Face-Kommunikation eingeführt, weil der Wunsch nach festen Beziehungen auch außerhalb des virtuellen Raums entsteht. Die Angst vor Sanktionen bei einem möglichen Treffen würde also von vornherein eine Selbstkontrolle bewirken (Schmidt, 2000: 19). Tatsächlich haben in der Befragung von Scherer und Wirth die Personen, die nur wenig „schummeln“, auch mehr Real-Life-Freunde im Chat gefunden als Personen, die mehr schummeln (Scherer/Wirth, 2002: 352). Warum aber lohnt sich die Disziplin auch für Menschen, die keinen Real-Life-Kontakt suchen, wenn keine Notwendigkeit für die Selbstbeherrschung besteht?

2. Die Sanktionen, die in eventuellen Real-Life-Kontakten zu befürchten sind, erscheinen abstrakt und von der aktuellen Kommunikation weit entfernt. Sanktionen von Identitätstäuschungen und Verletzung von Kommunikationsregeln lassen sich aber auch viel unmittelbarer finden: Wir haben oben die Analogie des Chattens mit dem Alltagsgespräch ausgeführt, so dass man von einer ähnlichen Selbstzweckhaftigkeit des Chattens ausgehen darf. Ein mögliches Ziel ist also das Chatten selbst, das den Teilnehmenden Vergnügen bereitet. Beim Alltagsgespräch ist die eigene Person stets Ausgangs- und Referenzpunkt. Verstellt man sich, ist dies nicht nur anstrengend, sondern bringt auch keinen Nutzen, da die Teilnehmer ja de facto nicht mit einem selbst, sondern mit der fingierten Person reden (Scherer/Wirth, 2002: 341). Auf der anderen Seite, wenn man als Chatter nun die potenziellen Möglichkeiten hinsichtlich Anonymität und Unverbindlichkeit ausnützt und sich so verhält, wie es im Real Life negative Konsequenzen hätte (z. B. andere beleidigt), wird die Kommunikation von Seiten der anderen Teilnehmenden abgebrochen. Damit ist allerdings auch der Chat beendet. In beiden Fällen ist das Resultat ein Verlust des Chat-Vergnügens. Nur wenn sich Chatter an die Regeln der konventionellen interpersonalen Kommunikation halten, können sie an der Kommunikation teilhaben und am Vergnügen, beim Chat „mitzuspielen“. Auch Höflich und Gebhardt interpretieren das Ziel des Chattens weniger instrumentell: Es scheint beim Chatten eben nicht in erster Linie darum zu gehen, ,auf stabile und über den Chat hinausgehende persönliche Beziehungen zurückgreifen zu können, sondern vielmehr darum, auf spielerische Art und Weise mit neuen Kommunikationsmöglichkeiten umzugehen" (Höflich/Gebhardt, 2001: 10).

\section{Das Spielmodell}

Den Chat als Spiel zu betrachten, kann nicht nur plausibel machen, warum sich die Chatter an grundlegende Umgangsformen auch in der anonymen Situation halten und in der Regel keine größeren Täuschungen über die eigene Person anstellen, sondern stellt auch eine Chance dar, unspezifische Motive der Chatnutzung wie Zeitvertreib und Unterhaltung zu konkretisieren.

Diesen Gedanken wollen wir nun in einem Spielmodell des Chattens ausbauen, das die Grundelemente konventioneller Spiele auf den Chat anwendet. Rieber (1996: 44 f.) definiert das Spiel als üblicherweise freiwillige Tätigkeit, die intrinsisch motiviert ist, d. h. ohne äußere Anreize ausgeführt wird, die ein aktives (oft physisches) Engagement 
erfordert, und die sich von anderen Tätigkeiten durch ihre „make-believe-quality“ unterscheidet und eine Art Scheinwelt aufbaut (vgl. ähnliche Definitionen in Pellegrini, 1995; Blanchard/Cheska 1985).

Linder/Roos/Victor (2001: 5) gehen darüber hinaus und sehen Regeln als konstitutive Elemente des Spiels. Die Scheinwelt, die vom Spielkontext aufgebaut wird, muss dabei in der Fantasie rekonstruiert werden: „In play, the imaginary situation permits a shift from a real world dominated by things and actions, to a world dominated by meaning" (Linder/Roos/Victor, 2001: 8). Ähnlich argumentiert Stephenson: „Characteristic of a game is its rules, its repetitions, its demand for fair play (...) the player has to be transported into a special place psychologically, if not physically as well“" (Stephenson, 1964: 369 f.).

Wie sind diese Merkmale nun auf den Chat anzuwenden? Spielort ist ein virtueller Raum, in den die Spieler sich psychologisch hineinversetzen, was teilweise durch die Benennung der Chats als Cafés oder Bars unterstützt wird. Zum Ablauf des Spiels gibt es zwei Arten von Regeln: Erstens existieren Spielregeln („rules“, Stephenson; „constitutive rules" Linder/Roos/Victor, 2001: 7), die die strukturellen Bedingungen und die formalen Abläufe des Spiels fixieren, wie sie etwa in der Spielanweisung bei konventionellen Spielen zu finden sind. Beim Chat sind solche Spielregeln in der grundlegenden Kommunikationsstruktur zu sehen (man meldet sich an, kann Beiträge liefern, kann sich in private Chats ausklinken), in den Netiquetten, der symbolischen Bedeutung von Emoticons, die das Fehlen von analogen Codes der Körpersprache und Intonation ausgleichen, und anderen standardisierten Gebräuchen (z. B. eine gerichtete Ansprache durch @Name). Zweitens gibt es „ungeschriebene Gesetze“, die individuelle und bewährte Spielstrategien umfassen, möglichst effizient zum Spielziel zu kommen, und die nicht festgeschrieben sind, sondern sich durch Erfahrung entwickeln („regulatory rules“ Linder/Roos/Victor, 2001: 7). Als Beispiel dafür kann man etwa bei Monopoly die Strategie nennen, sich auf die teuren Straßen zu konzentrieren, weil diese ertragreicher sind.

Wir unterscheiden zusätzlich zwei Arten von Zielen: Zum einen gibt es das Spielziel, das ausdrückt, wonach die Handlungen innerhalb des Spielsystems ausgerichtet sind und wann man gewonnen hat; bei Mensch-ärgere-dich-nicht bestünde das Spielziel darin, alle vier Figuren nach Hause zu bringen. Zum anderen gibt es das Metaziel, das die Ziele der Teilhabe am Spiel ausdrückt, wie etwa Unterhaltung, Ablenkung, Zeitvertreib (Weinreich, 1997: 117), und das selbstzweckhafte Moment jeden Spiels beinhaltet - das Vergnügen am Spielprozess.

Spielhandlungen bestehen aus dem Lesen und Schreiben von Threads, die nach einer Rahmung der aktuellen Situation geplant und ausgeführt werden. In die Rahmung fließen der Chat-Rahmen, die Spielregeln, die Strategien sowie Spiel- und Metaziel ein. Mit der Darstellung des Modells (s. Abb. 1) werden die noch erklärungsbedürftigen Aspekte offenkundig: Während die Spielrollen (Spieler/Spielleiter), das Metaziel und die Spielregeln gut aus bisheriger Forschung abgeleitet werden können, bleiben Spielziel und Spielstrategien noch im Dunkeln und sollen daher Gegenstand unserer empirischen Untersuchung sein.

\section{Untersuchungsaufbau}

Spielziele und -strategien sind in den Prozess des Chattens eingebettet. Da nicht angenommen werden kann, dass diese Aspekte außerhalb der Chat-Situation von Chattern rekonstruiert werden können, erscheint die Beobachtung des Chat-Prozesses die geeignete Methode zu sein. Daher kam hier eine Kombination aus Beobachtung und Lautem 


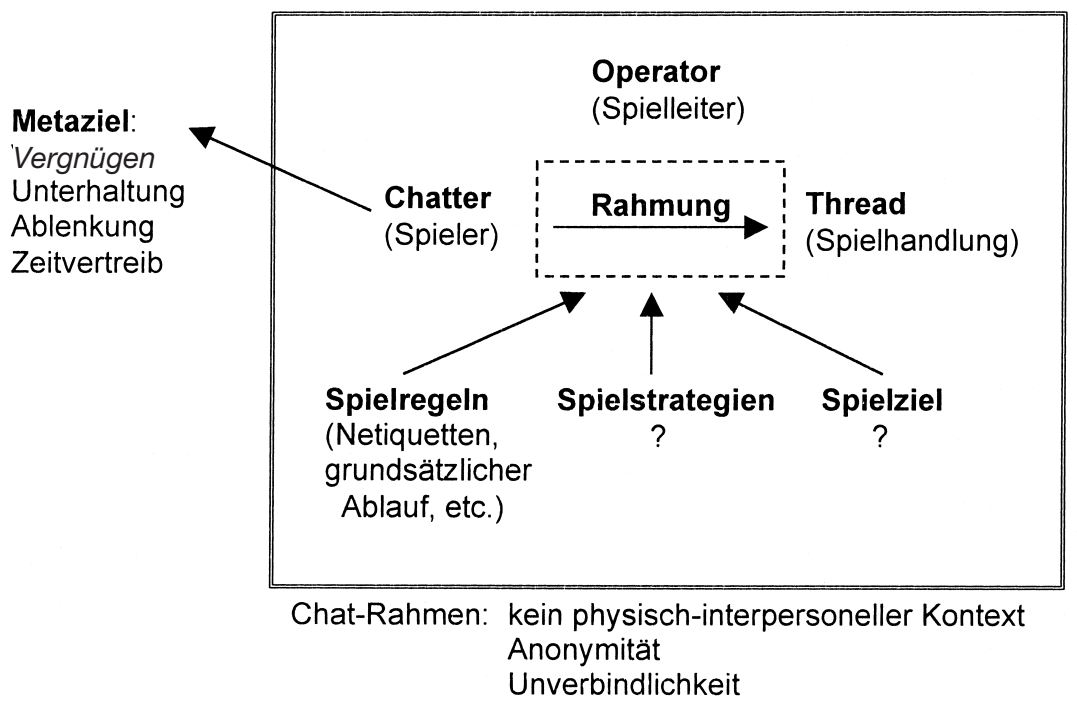

Denken zur Anwendung. Zwölf freiwillige Probanden ${ }^{3}$, regelmäßige Chatter, wurden bei einer ihrer Chatsitzungen für 45 Minuten beobachtet. ${ }^{4}$ Dabei wurden sie aufgefordert, alles laut auszusprechen, was ihnen gerade durch den Kopf geht (Lautes Denken, Ericsson/Simon, 1993; Bilandzic/Trapp, 2000). Eine viertelstündige Aufwärmphase, deren Daten nicht zur Auswertung verwendet wurden, ging der eigentlichen Beobachtung voraus. Das Chatgespräch sowie die verbalisierten Gedanken der Probanden wurden auf Video aufgezeichnet und später transkribiert. Die Probanden wurden über Kontaktpersonen aus dem weiteren Bekanntenkreis der Erstautorin rekrutiert, die den Kontakt zu ihnen bekannten regelmäßigen Chattern herstellten. Die relativ aufwändige Beobachtung mit einer Dauer von insgesamt zwei Stunden legte eine solche Vorgehensweise nahe, nicht zuletzt, um überhaupt Teilnehmer zu gewinnen. Die Bereitschaft zur Teilnahme war über die Vermittlung durch bekannte Personen gesichert, war aber gleichzeitig nicht von einem engen sozialen Verhältnis belastet. Die Probanden wurden angewiesen, ihre angestammten und damit vertrauten Chats aufzusuchen, um eine möglichst natürliche Situation vorzufinden. Dies hatte auf der anderen Seite die Konsequenz, dass verschiedene Chats besucht wurden und bestimmte Aspekte der Situation, wie z. B. die Vertrautheit mit den anderen Chattern, nicht kontrolliert werden konnten. Dies wurde aber in Kauf genommen, um die Chatter in der Umgebung zu beobachten, die sie ge-

3 Acht Männer und vier Frauen im Alter von 19 bis 34 Jahren, was dem ,Mainstream` der Online-Nutzer entspricht.

4 Die Daten wurden ursprünglich im Rahmen der Magisterarbeit der Erstautorin an der Universität München erhoben. Es wurde dafür auch ein Leitfadeninterview im Anschluss an das Laute Denken durchgeführt, in dem die Ansichten der Probanden zum Chat noch einmal vertieft wurden. 
wöhnt waren. Wollte man untersuchen, welchen Einfluss die Art des Chats oder der Bekanntheitsgrad der Chatter untereinander hat, müsste man gezielt diese Aspekte variieren, was aber hier nicht im Mittelpunkt stand und sicherlich eine größere Stichprobe erfordert hätte. Die Vorteile des Lauten Denkens liegen darin, dass die Prozesse, die beim User während der Nutzung ablaufen, nachgezeichnet werden können. Nach der Aufwärmphase haben sich die Probanden auch in der Regel an die Versuchssituation gewöhnt und sprechen ihre Gedanken automatisch aus ohne darüber nachzudenken, was sie sagen. Die Grenzen des Lauten Denkens liegen aber ganz klar darin, dass nur bewusste psychische Vorgänge überhaupt eine Chance haben, ausgesprochen zu werden; unbewusste Vorgänge, Gefühle, Wahrnehmungen bleiben außen vor. Die Gesamtheit aller psychischen Prozesse wird man daher nicht erfassen können. Für unsere Zwecke war die Methode jedoch ausreichend, da es um Strategien und Ziele ging, von denen angenommen werden kann, dass sie den Usern zumindest während des Chattens bewusst sind (wenn auch nicht in einem analytischen Sinne, dass sie als Spiel identifiziert werden).

Die Auswertung wurde folgendermaßen durchgeführt: Die interessierenden Elemente unseres Spielmodells, Spielziele und -strategien, wurden als heuristische Kategorien an das Material herangetragen. Die heuristischen Kategorien stellen hier begrifflich definierte, aber empirisch noch nicht ausgebaute (d. h. in ihren genauen Ausprägungen noch unbekannte) Konzepte dar, die als Selektionskriterium dienen, um die interessierenden Stellen des Think-aloud-Transkripts für eine weitere Analyse auszuwählen. ${ }^{5}$ Die heuristischen Kategorien wurden folgendermaßen definiert:

- Spielziel: Thematisieren von Absichten, Wünschen und Gratifikationen, die sich auf den Chat selbst beziehen.

- Ungeschriebene Gesetze: Thematisieren eigener und fremder Strategien des Chattens und Äußerung von Handlungsregeln.

Stellen des Transkripts, die mit Hilfe dieser Definitionen identifiziert werden konnten, wurden einer Kategorienbildung unterzogen. Dazu wurde das Material zunächst mit Hilfe der theoretisch-heuristischen Segmentierung in einzelne gleichwertige Teile zerlegt, um eine Basis für die folgende systematische Inhaltsverdichtung zu erhalten (zum Verfahren siehe Bilandzic/Koschel/Scheufele, 2001). Dieses Verfahren ist geeignet, die sonst dominierende induktive Vorgehensweise bei der qualitativen Inhaltsanalyse intersubjektiv nachvollziehbarer zu machen, indem theoretisches Vorwissen explizit in die Kategorienbildung einfließt. Dieses theoretische Vorwissen ist durch die Rahmenanalyse und das Spielmodell abgesteckt: Im Zentrum des Spiels steht eine Interaktion zwischen Chattern über medial vermittelte Threads. Das heißt, dass jede Äußerung im Lauten Denken als Anlass oder Gegenstand einen Thread oder einen Chatter haben wird. Daher dienen Verweise auf eigene und fremde Threads sowie Verweise auf andere Chatter als Segmentierungskriterien. Wird ein neuer Verweis genannt, so beginnt auch ein neues Segment. Das weitere Vorgehen hat die Kategoriebildung zum Ziel (Bilandzic/Koschel/Scheufele, 2001: 108 ff.; ähnlich Mayring, 1997): Zunächst werden die Segmente sprachlich und inhaltlich reduziert und die Kernaussagen einheitlich und allgemein reformuliert. Daraufhin werden über die Segmente hinweg Klassen ähnlicher Kernaussagen gebündelt. Schließlich werden die entstandenen Klassen mit dem theoretischen Vorwissen verknüpft und definiert. In der Ergebnisdarstellung werden die Er-

5 Vgl. ähnliche Konzepte heuristischer Leitideen bei der Theoriekonstruktion: Blumer, 1954; zur Übersicht siehe Kelle, 1997: 225 ff. 
kenntnisse anhand von Beispielen interpretiert. Dieses Auswertungsverfahren ist gezielt so konstruiert, den Prozess des Spielens näher zu untersuchen; andere Aspekte müssen damit zugunsten dieser gezielten Analyse herausfallen (z. B. die Gewichtung des Spielrahmens im Vergleich zu anderen Rahmungen).

\section{Ergebnisse}

\subsection{Spielziel I: Entlarvung der Kommunikationspartner}

Als ein wesentliches Ziel des Kommunikationsspiels Chat hat sich die Entlarvung der Schlüsselmerkmale der Kommunikationspartner herauskristallisiert, die in drei Schritten abläuft. Wie bereits erwähnt, sind mit Schlüsselmerkmalen Grundinformationen über einen Menschen gemeint, also Merkmale wie Alter, Geschlecht, Aussehen etc., und nicht die Persönlichkeit oder eine „wahre“ Persönlichkeit.

Schritt 1: Zunächst versuchen die Chatter, sich über den Nickname einen ersten Eindruck von den anderen Kommunikationsteilnehmern zu verschaffen. Durch den Nickname wird eine Erwartung generiert, und oft beginnt die Kommunikation mit dem Ziel, diese Erwartung zu überprüfen. So spricht Sofa-Surfer1 DynamiteD an, weil: „DynamiteD, das klingt für mich ziemlich prollig und deshalb find ich's interessant, was der so von sich gibt“ und Donk spricht Blödel an: „Blödel ist doch ein super Name...das ist immer sehr aussagekräftig, wenn sich die Leute solche Namen geben.“ Nicknames können darüber hinaus auch den ersten Gesprächsstoff einer Kommunikation liefern. So entspinnt sich um den Namen Sofa-Surfer ein ganzes Gespräch; der Nickname wird zum Selbstläufer (Klemm/Graner, 2000: 163).

Schritt 2: Anschließend geht es darum, die Schlüsselmerkmale der Gesprächspartner schrittweise zu enttarnen und etwa Geschlecht, Alter, Wohnort und Beruf in Erfahrung zu bringen. Werden die Vorteile der computervermittelten Kommunikation oft darin gesehen, dass herkömmliche Identitätskategorien und Statusmerkmale in den Hintergrund treten, stellte sich bei unseren Probanden heraus, dass gerade nach diesen im Verlauf des Chats geforscht wird, um das Bild der Gesprächspartner zu vervollständigen.

Für Alasta900 ist z. B. das Alter so wichtig, dass er danach entscheidet, ob das Gespräch überhaupt fortgesetzt werden kann: „Ich frag immer, wie alt ist er oder sie. Weil, wenn sie so jung sind, ich mach' nicht weiter." Regenfrau ist insgesamt durch die Enttarnungsversuche ziemlich ungehalten: „Also, eigentlich wäre der jetzt normalerweise schon wieder voll abgeschossen, erste Frage: männlich oder weiblich, zweite Frage: Alter...pf...wahrscheinlich kommt dann noch Größe: Hurra!!“ Regenfran weist damit nicht nur auf die mangelnde Originalität ihres Gegenübers hin, sondern kritisiert vor allem die Plumpheit seiner Spielpraxis - wer den Prozess der Entlarvung nicht geistreich ausgestaltet, ist ein Spielverderber.

Nicht nur die direkte Frage an die Mitchatter kann Aufschluss geben über ihre Person; es werden auch fleißig Indizien gesammelt und Schlussfolgerungen gezogen. SofaSurfer2 überlegt bezüglich des Wohnortes von Virus: „...wenn er zum Union-Move will, dann kann er nicht so weit weg wohnen!“ Sofa-Surfer1 stellt fest: „... sind sowieso viel mehr Männer unterwegs als Frauen ... Was sie schreiben, bzw. von der Art und Weise her, wie sie es schreiben“ und zieht damit aus den Textbeiträgen selbst Rückschlüsse auf das Geschlecht (vgl. auch die Ergebnisse von Bahl, 1997). Alice fragt: „What time is it over there?“, denn es „,ist immer interessant, wenn man sich überlegt, wie viel Uhr es bei denen ist, es ist jetzt irgendwie neun in der Früh, davon kann man immer schließen, was des für Leute sind ...". 
Auch die Visitenkarten, jederzeit abrufbare Selbstbeschreibungen, können als Hinweise dienen. MartinK, Donk, Sofa-Surfer 2 und Airnesto nutzen diese Möglichkeit. „Ich schau mir die Leute immer vorher an“, äußert MartinK und findet es „echt gemein“, dass die meisten nichts in ihre Karte eingetragen haben. ${ }^{6}$

Schritt 3: Neben der Enttarnung der Schlüsselmerkmale ihrer Kommunikationspartner sind die Chatter außerdem damit beschäftigt, die Glaubwürdigkeit der Threads zu prüfen, um dadurch mögliche Täuschungen zu entlarven. Zwar haben nur vier Probanden während der Beobachtung über sich selbst Unwahrheiten erzählt; es waren sich allerdings alle der Tatsache bewusst, dass sie selbst jederzeit , angeschwindelt ${ }^{`}$ werden können.

Groemucs Chat-Partnerin AnnaS hat sich zwar als 15-jähriges Mädchen geoutet, aber: „Ja, wer weiß, vielleicht ist des irgendwie ein 30-Jähriger!“ Und bei JerryLee meint er: „Ich schätze sie mal als Studentin ein ... oder als 80-jährigen fetten Opa, hahaha!“. Auf die Beschreibung von Manuel, dass er 24 Jahre, jung und sportlich sei, meint Idefix nur: „Ja, wer hätte das nicht gern?“

Zur Prüfung der Glaubwürdigkeit werden Kontrollfragen gestellt. Adonis hat Dia bereits gesagt, dass er $25 \mathrm{sei}$, aber sie stellt noch mal die Frage nach seinem Alter. Auch Regenfrau nutzt die Möglichkeit, noch mal nachzufragen: „... in Regensburg war er an den Wochenenden ... mal schauen, ob das so stimmt ...". Groemuc ist selbst Subjekt von Kontrollfragen von AnnaS: „... es kann sein, dass sie mich jetzt testen will, wer ich bin“. Das ständige Hinterfragen der Glaubwürdigkeit und das Stellen von Kontrollfragen sind also die Strategien, wie sich Chatter mit der Möglichkeit arrangieren, jederzeit einer Täuschung ausgeliefert zu sein (Höflich, 1999).

\subsection{Spielziel II: Steuerung der Selbstdarstellung}

Die unweigerliche andere Seite der Entlarvung ist es, die Gesprächspartner mit Hinweisen zur eigenen Person zu beliefern und damit die Selbstdarstellung zu steuern. SuttonSmith (1997) beschreibt das „play as self“ als eine Variante konventioneller Spiele, die das Sammeln von Lebenserfahrungen über das Ich in den Vordergrund stellt.

Eine Täuschung über die eigene Person kann im Chat dazu dienen, die Kommunikationschancen zu verbessern. Alasta900 nutzt die Möglichkeit, sich bei Serbiangirl-yu ein paar Jahre jünger zu machen. Er musste nämlich bei der 18-jährigen Pretty-Orange-Katt feststellen, nachdem er sein Alter (29 Jahre) verraten hatte: „Hm, sie antwortet nicht, wahrscheinlich ist sie enttäuscht, weil ich elf Jahre älter bin!“"Als er von Serbiangirl-yu schließlich nach seinem Alter gefragt wird, meint er: „Also, sag ich ein bisschen jünger.“ Und so werden aus 29 ganz einfach 25 Jahre. Sofa-Surfer1 sagt: „Mal ein bisschen provozieren, mal schauen, vielleicht steigt er drauf ein!" als er auf die Frage von Visitor4219: „bin aus Augsburg und suche wwwwww!“ antwortet:,allo Schwabe, hier ist w“. Allerdings kommt Sofa-Surfer1 nicht dazu, seinen Geschlechtswechsel weiter-

6 Man kann nur spekulieren, warum diese Möglichkeit eher selten genutzt wird. Mit dem Spielmodell könnte man gut erklären, warum Visitenkarten unattraktiv sind: Das Entlarvungsspiel und das Spiel um die eigene Identität wären jäh beendet, könnte man gleich nachlesen, wer sich hinter welchem Nick verbirgt. Somit wäre die Visitenkarte kontraproduktiv zum Spielziel. Freilich sind auch andere Gründe denkbar, etwa die grundsätzliche Abneigung der User, persönliche Informationen irgendwo im Netz zu fixieren. 
zuspinnen, da das Gespräch nicht weitergeführt wird. In beiden Beispielen wird explizit die Absicht geäußert, mit der Selbstmodifikation die eigenen Kontaktmöglichkeiten zu verbessern.

Eine weitere Absicht, die hinter der Modifikation der Schlüsselmerkmale steckt, ist die, das Spiel um die Identität spannender und interessanter zu machen. Die unverhohlene Suche anderer Chatteilnehmer nach weiblichen Chatterinnen treibt Donk dazu, sich zu verstellen und einen eifrigen Sucher mit ambivalenten Hinweisen zu foppen: Auf die Frage von Spookey, ob er männlich oder weiblich sei und wo er herkomme, meint Donk: „Jaaa, das würdest jetzt gerne wissen, Spookey, ha, lassen wir ihn mal im Zweifel ... ,ich komme aus Bayern'... jetzt denkt er sicherlich, er hat es mit einer netten Münchnerin zu tun.“ Abermals fragt Spookey: „m o w? donk?“ „Schauen wir mal ... haha, spielen wir mal die doofe Frau ... ja, jetzt geben wir ihm einfach mal ein bisschen die Blondine ... Jetzt frag ich ihn doch mal, ob er solo ist ... der arme Kerl wird immer mehr denken, er hat's mit einer Frau zu tun!“ Schließlich macht Donk jedoch einen Fehler, der zu seiner Entlarvung führt: Er spricht Lolita an, woraus Spookey schließt: „Donk ist also m!“ Daraufhin verschwindet Spookey aus der Unterhaltung. Donks Kommentar: „Ich glaub, Spookey ist jetzt beleidigt ... weil er gemerkt hat, dass ich gar keine Frau bin." Eine einfache Antwort auf Spookeys Frage hätte die Konversation vermutlich sofort im Keim erstickt. Auf diese Weise hat Donk ein Rätselspiel entsponnen, das seinen Reiz durch die verwirrten Reaktionen Spookeys auf die ambivalenten Hinweise erhält.

Groemuc tritt als Einziger längere Zeit als eine andere Person auf. Er verändert Alter, Wohnort und Lebenssituation. An der Kommunikation mit AnnaS zeigt sich deutlich, welche Schwierigkeiten dabei auftauchen können. „Schauen wir mal, was das wird, jetzt bin ich 17!“ Die Frage nach seinem Wohnort nimmt Groemuc noch ganz gelassen: „Hm, ja wo komm ich denn her?" und entscheidet sich für Neuhausen. Als AnnaS aber wissen will, auf welcher Schule er sei, wird es schwieriger: „Was für eine Schule? Scheiße, was für eine Schule????“ Da die Schulzeit doch schon zu lange her ist, gibt er schließlich an, eine Lehre zu machen. Doch plötzlich weiß er nicht mehr, welches Alter er angegeben hatte: „... sag mal, wie alt bin ich denn eigentlich - 17, oder?" Als Kucki auftaucht und fragt: "Suche netten ihn so um die 30" freut sich Groemuc und will sich bemerkbar machen, muss aber dann feststellen: „Ah, ne, jetzt kann ich ja nicht reingehen ... ich würde mich ja voll outen, haha!" Schließlich wird die Rolle des 17-jährigen Azubis doch etwas anstrengend. „... ich muss mich jetzt da irgendwie entlang hangeln und 'ne längere Konversation führen, ohne dass, äh, ohne meine Identität preiszugeben, und sie dann irgendwie zu verärgern. “ Dem lockeren und entspannten Chatten bereitet eine frei erfundene Identität ein jähes Ende: Es ist anstrengend, die neue Person konsistent und dauerhaft zu spielen. Grenzen werden auch durch das Verantwortungsgefühl gesetzt; der Gesprächspartner soll nicht verletzt werden, der Chatter will selbst aber auch weiterhin im Gespräch integriert bleiben. Er hat sich durch das Darstellen einer anderen Person aber Kommunikationschancen verbaut, die seine eigene Person mehr interessiert hätten.

Weder bei anderen noch bei sich selbst wird die Variation des Selbst als negativ angesehen: Die Verstellung der anderen Chatter wird ständig mitgedacht und bringt im Sinne von Rätselraten auch Spielvergnügen. Mit der eigenen Verstellung kann man auf der anderen Seite den Verlauf des Spiels steuern. Sofa-Surfer2 bringt dies auf den Punkt: „Das ist Chatten. Das ist beim Chatten das A und O. Es ist ja klar, dass jeder sich falsch gibt. Das darf man nicht negativ sehen. Aber Chatten ist, sich in einer anderen Rolle ausgeben." 


\subsection{Spielstrategie: Kommunikationsgeschicklichkeit}

Das Beherrschen der Kommunikation in inhaltlicher und technischer Hinsicht trennt Könner von Nicht-Könnern im Chat-Spiel. Schnelligkeit und ständige Aktivität sowie Schlagfertigkeit sind unabdingbar, um Kommunikationspartner zu finden und zu halten. Die taktische Planung von Kontaktaufnahme und Gespräch dient ebenfalls diesem Zweck. Schließlich müssen Chatter auch mit der Unübersichtlichkeit und Komplexität der Chatkommunikation fertig werden.

Schnelligkeit und ständige Aktivität. Während man sich in direkten Gesprächen mit einem freundlichen Nicken aus der Affäre ziehen kann, oder auch einfach mal schweigt, gelten im Netz andere Maßstäbe: „Du darfst hier nicht inaktiv bleiben. Des geht total schnell vorbei an dir, der ganze Spaß ...“ (Groemuc). Was droht, ist eine der härtesten Sanktionen im Chat, nämlich ignoriert zu werden („Höchststrafe“, Klemm/Graner, 2000: 164). „Also, im Prinzip bin ich jetzt offline“, bemerkt Groemuc schließlich, als sich seine Gesprächspartnerin nicht mehr meldet und er auch keine anderen Kontakte knüpfen kann. Nicht nur eine ständige Aktivität ist wichtig, auch die Schnelligkeit einer Reaktion auf andere Ansprachen ist notwendig, weil sich sonst der Gesprächspartner abwendet oder jemand anders den Thread aufgreift. Sofa-Surfer 2 äußert etwas resigniert: „... ach, der Angel hat da schon geantwortet, der war schneller!“ Auch MartinK verpasst seine Chance: „Uha, schon vorbei ... da muss man viel zu schnell sein, sonst ist es langweilig!“.

Schlagfertigkeit und Originalität. Nicht nur zahlreich und prompt sollen die Äußerungen sein, sondern auch noch schlagfertig und originell, um im Gewirr der Threads Aufmerksamkeit zu erregen und Gesprächspartner zu gewinnen. Zwar sind herkömmliche Statusmerkmale ausgeblendet, wodurch alle Teilnehmer zunächst einmal die gleichen Kommunikationschancen haben, jedoch werden nun Schlagfertigkeit und Originalität ausschlaggebend für eine Aufnahme ins Gespräch. So werden eigene Bemerkungen mit diesem Zwang zur Originalität begründet. Auf die Frage, auf welchem Sofa Sofa-Surfer1 surft, entgegnet er: ,Irisches Schafswollsofa auf Gummistelzen’ - „um aufzufallen und einfach 'ne interessante Antwort zu geben, die ein bisschen abgefahren klingt“. Den Abbruch eines Gesprächs erklärt Regenfrau mit der mangelnden Originalität ihres virtuellen Gegenübers Puffy: „Na, ja, Puffy ist nicht so der Brüller...“ und ignoriert seine wiederholten Kontaktversuche. Sofa-Surfer 2 freut sich, als er herausfindet, dass sich hinter dem Pseudonym Roudgirl zwei Personen verstecken: „Super, zwei sind noch besser. Doppelt so viele Ideen!“ Nicht nur die Kommunikate selbst transportieren Originalität; bereits der Nickname verschafft einen ersten Eindruck vom „OriginalitätsPotenzial“ des Chatters und kann auch Anlass für eine Kontaktaufnahme sein: „,Webgoofy ‘ - das ist ja geil... ,Hi Webgoofy‘...das muss man ja belohnen!“, meint Donk und belohnt ihn mit der Währung des Chat: mit Aufmerksamkeit.

Strategien der Kontaktaufnahme und Kommunikationstaktiken. Das Problem der Kontaktaufnahme wird von den Chattern ständig reflektiert: „Bis man da direkt einen richtig drankriegt, das ist schwierig... na super, hey, was mach ich heute falsch? Warum will keiner mit mir schreiben, verdammt noch mal?“ oder „Keiner antwortet mir, das ist ja gemein!“. Sofa-Surfer2 bemerkt schließlich: „Ich glaub, es ist komplizierter, ein Gespräch im Chat anzufangen als im wirklichen Leben, haha!“.

Aus dem Zwang nach Schlagfertigkeit, Originalität und Auffälligkeit entwickeln sie individuelle Kommunikationstaktiken, die von direkten Fragen an einzelne Chatter über gehäufte und schnelle Antworten, Provokationen bis hin zu Flüstermails reichen (Sofa-Surfer1 flüstert ein paar Chattern einfach „Alles Gute zum Geburtstag!“zu). Dia, 
Airnesto und Regenfrau gehen nach einem Ausschlussprinzip vor: Viele ansprechen, damit vielleicht ein paar übrig bleiben und loggen sich dafür gleichzeitig in mehreren Foren ein. Auch der Nickname kann über eine Kontaktaufnahme entscheiden: Regenfrau wird erst angesprochen, nachdem sie ihren ursprünglichen Nick Loopa ändert und kommentiert dies: „... irgendwas mit ,Frau' hintendran... ich denke, dann wird man eher angelabert." Was im echten Leben die Kleidung und die Frisur ist, ist im Chat die Schrift: Will man auffallen, so legt man sich eine auffällige Schrift in einer auffälligen Farbe zu.

Textuelle Komplexität. Neben der inhaltlichen Gewandtheit ist auch Konzentrationsfähigkeit gefordert, um die verschiedenen parallelen „kommunikativen Baustellen“ im Auge zu behalten und den Faden in der jeweiligen Unterhaltung nicht zu verlieren. Sofa-Surfer 2 formuliert dies folgendermaßen: „Also, das ist irgendwie doof, wenn man mehrere Leute gleichzeitig am Start hat ... dann kennt man sich überhaupt nicht mehr aus.“ Auch Regenfrau kämpft mit diesem Problem und gibt irgendwann zu: „Was? Also irgendwie habe ich jetzt langsam den Überblick verloren ... Jetzt wird es echt stressig! ... Äh, was war??“ Das Wirr-Warr an Kommunikaten, die technisch bedingt in einer beliebigen Reihenfolge auf dem Bildschirm erscheinen, erschwert die Orientierung gerade bei mehreren parallelen Gesprächen. Das Beherrschen dieser textuellen Komplexität (z. B. Jakobs, 1998: 196) ist aber unabdingbare Voraussetzung für das Spiel. So müssen Chatter darauf achten, keine Antworten zu verpassen, während sie ihre Repliken tippen. Donk ist z. B. noch so mit Spookey beschäftigt, dass ihm schließlich einfällt: „... jetzt hab ich nicht mal gesehen, ob mir die Lolita vorher geantwortet hat." Dia fragt Loverman, ob es ihm jetzt die Sprache verschlagen hätte, weil er nicht mehr antworten würde. Doch dann stellt sie fest: „Ah, da hat er doch schon geschrieben, peinlich, haha.“

Die Aufgabe, mehrere Gesprächspartner gleichzeitig zu bedienen, führt regelmäßig zu solchen Pannen, weshalb sich Chatter damit abgefunden und ihre Kommunikationserwartungen dementsprechend angepasst haben: „Hm, man überliest immer irgendwas ...", bemerkt Dia; als Idefix keine Antwort auf seine Frage bekommt und feststellt, dass BlueScreen die Frage überhaupt nicht bemerkt hat, meint auch er ganz gelassen: „Na gut, dann muss man halt noch mal fragen.“

Da eine erfolgreiche Kommunikation Voraussetzung ist, um überhaupt am Spiel teilzunehmen, bestimmen diese Geschicklichkeiten über Erfolg und Misserfolg einer Kommunikation und damit über das Erreichen von Spiel- und Metaziel. Mehr noch, nur Kenntnis über die Identität des anderen zu erlangen, heißt nicht ohne weiteres, auch das Metaziel „Vergnügen“ zu erreichen: Der Weg dorthin ist entscheidend, die Freude an der eigenen Geschicklichkeit und der der anderen Chatter. Nicht umsonst hatte Regenfrau ärgerlich reagiert, als jemand versucht hat, diesen Weg plump abzukürzen. Wir können damit die vorher formulierten Spielziele präzisieren: Entlarvung und Selbstdarstellung sind die konsensuellen Leitmotive des Spielrahmens ${ }^{7}$ (Abb. 2). Es geht jedoch nicht einfach nur darum, möglichst schnell Namen und Basisinformationen über den Partner herauszufinden; sonst würden ja die Visitenkarte oder die direkte Frage nach soziodemografischen Merkmalen die rationellsten Wege zur Erreichung des Spielziels darstellen. Doch das eine wird kaum genutzt, das andere entlarvt den bemühten Chatter als Di-

7 Goffman beschreibt den Prozess der Situationsdefinition als Bühne für ein »Informationsspiel « bzw. als endlosen »Kreislauf von Verheimlichungen, Entdeckungen, falscher Enthüllungen und Wiederentdeckung «(Goffman 1969: 12) - und fasst gleichzeitig genau die Prozesse zusammen, die auch beim Chat-Spiel beobachtet werden können. 
lettanten. Die unterhaltsame und originelle Ausgestaltung des Wegs dorthin macht das Spiel aus.

\section{Abbildung 2: Spielmodell des Chattens}

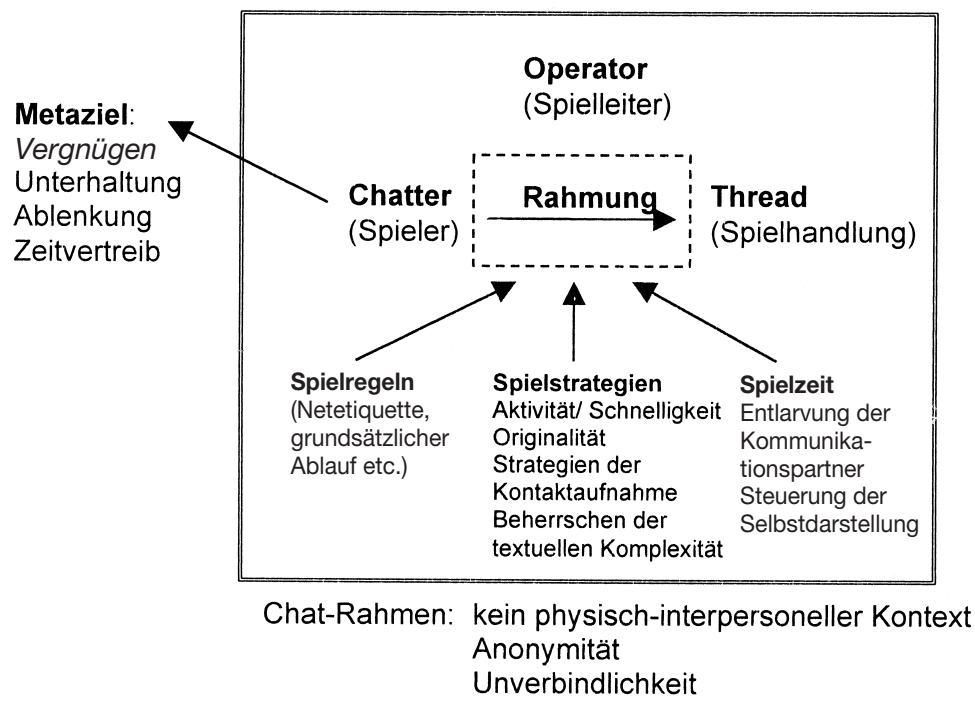

6. Fazit

Betrachtet man den Chat als Kommunikationsspiel, wird klar, warum sich die Mehrheit der Teilnehmer an die Regeln konventioneller Kommunikation hält und die Möglichkeiten der Anonymität und Unverbindlichkeit nur selten zu langfristigen und schwer wiegenden Täuschungen missbraucht: Wer sich nicht an die Spielregeln hält, wird ignoriert und muss auf das Spielvergnügen verzichten. Hingegen gehören kleinere und kurzfristige Täuschungen fast schon zum Spiel dazu, jeder rechnet bei anderen damit und macht es selbst, um das Spiel voranzubringen. In den Gedankenprotokollen der Chatter haben wir die Entlarvung des Gegenübers und die Steuerung der Selbstdarstellung als Spielziele identifizieren können. Nicht aber die bloße Kenntnis der fremden Identität oder eine möglichst positive Selbstdarstellung ist wichtig, sondern die kreative Ausgestaltung des Prozesses, die mit der Kommunikationsgeschicklichkeit als Spielstrategie steht und fällt. Ein plumpes Nachfragen „m o w? "verärgert und verprellt die Gesprächspartner. Was das Spielvergnügen ausmacht, ist ein geschicktes Anpirschen an den Anderen, das Sammeln von Indizien und ein scharfsinniges Schlussfolgern. Man kann also sagen: Der Weg ist das Spielziel.

Nur wer diese Kommunikationsgeschicklichkeit - ständige Aktivität, Originalität, taktisches Vorgehen und Meistern der textuellen Komplexität - beherrscht, wird von anderen Chattern mit Aufmerksamkeit belohnt und „darf mitspielen“. Dieses Vergnügen am eigenen Witz und Scharfsinn findet man natürlich in vielen Spielen wieder; wo es aber besonders prominent ist und wo sich eine Parallele zum Chat geradezu aufdrängt, ist die Scherzkommunikation im privaten Kontext: Treffen sich Freunde oder Bekann- 
te, um zusammen zu plaudern, ist zu beobachten, dass die Ernsthaftigkeit gelockert wird, kreativ mit der Sprache umgegangen wird, schließlich auch gefrotzelt, gewitzelt und fantasiert wird (Hartung, 1996: 109). Diese Art der Kommunikation ist nicht nur als Hilfe in der Konfliktbearbeitung und Lebensbewältigung zu sehen, „diese Funktionen ... [können] zurücktreten ..., um der puren Lust an Sprache, Phantasie und Kreativität Platz zu machen“ und „oft wird nicht die scherzhafte Modalität instrumentalisiert, um soziale Probleme zu lösen, sondern soziale Probleme werden instrumentalisiert, um Scherzkommunikation so unterhaltsam wie möglich zu machen" (Hartung, 1996: 109). Vor allem die spielerische Provokation (teasing; Günther, 1996) hat den Sprung in die virtuelle Welt geschafft. Der Unterschied zur Real-Life-Scherzkommunikation liegt darin, dass die Gesprächspartner sich nicht unbedingt kennen. Sie verfügen insofern auch nicht ohne weiteres über einen gemeinsamen Erfahrungsschatz, aus dem sie für ihre Kommunikation schöpfen können. Das wiederum bedeutet, dass passende Partner erst ausgelotet werden müssen und es manchmal zwangsläufig zu Startschwierigkeiten kommt. Gleichzeitig ist der geringe Bekanntheitsgrad auch der Grund, warum sich die Chatter in der Regel an Gebote der Höflichkeit halten und warum sie auch ständig reflektieren, welche Handlungen welche Chatter beleidigen könnten: Ist eine „grundsätzliche Gutwilligkeit des Verhältnisses“ (Hartung, 1996: 112) nicht sicher, und das ist bei jungen Bekanntschaften nie der Fall, müssen die Spieler umso sensibler darauf achten, dass ihre Äußerungen nicht als böswillig missverstanden werden. Wenn nämlich diese Vertrauensgrundlage nicht vorhanden ist, ist auch eine spielerische Konversation nicht mehr möglich, weil dann scherzhafte Äußerungen als ernst gemeinte Angriffe gedeutet werden können (Hartung, 1996: 112) - diese Erkenntnis zur Scherzkommunikation als einer Form der Alltagskommunikation kann leicht auf den Chat übertragen werden.

Im Chat findet spielerische Alltagskommunikation zwar statt. Chatkommunikation ist aber mehr als nur computervermittelte Alltagskommunikation, und zwar durch zwei Aspekte: Erstens ist der Chat ein institutionalisiertes Forum für Spiel; d. h. es ist jederzeit für jeden verfügbar. Das hat unmittelbare Konsequenzen auf seinen Gebrauch: Man muss sich nicht verabreden, man hat gewisse Sicherheit, dass immer jemand da ist, und es ist normal und erwünscht, mit Menschen in Kontakt zu treten und auch Fremde anzusprechen (im Gegensatz zum Marktplatz).

Zweitens kann der Chat als „social laboratory“ betrachtet werden, in dem Handlungsweisen, Rollenkonzepte und die Reaktionen darauf gewissermaßen getestet werden können, wobei als Grenzen dieses »play as self « die allgemeinen Umgangsformen fungieren. Dieses Ausprobieren von Möglichkeiten ohne reale Sanktionen ist ein Charakteristikum von Spielen allgemein: ,in play we are able to control the content, the roles and the outcomes while experiencing attached emotional experiences. We explore and discover, trying out a variety of different styles of communication, enacting different roles, breaking out of the constraining confinements and limitations of everyday real life, testing the potential of diverse dramatic approaches, the free expression of feelings, or experimenting the desirability and the acceptability of social behaviors." (Linder/ Roos/Victor 2001: 13) Die Institutionalisierung und die Social-Laboratory-Eigenschaft unterscheiden den Chat von gewöhnlicher Alltagskommunikation und unterstreichen seinen Spielcharakter.

Der Spielrahmen steht natürlich nur für diejenigen Chatter im Vordergrund, die den Chat aus den von uns eingangs „unspezifisch“ genannten Motiven heraus nutzen. Wird der Chat aus Kontaktmotiven heraus genutzt und dementsprechend als Kontaktrahmen betrachtet, dürften andere Handlungsziele im Vordergrund stehen, z. B. das Herausfinden von E-Mail-Adresse und Telefonnummer oder die Vereinbarung eines Real-Life- 
Treffens. In einem Informationsrahmen hingegen könnte die beste Kneipe der Stadt oder der Termin der nächsten Love-Parade erfragt werden. In einem Therapierahmen würde man ernsthafte Probleme und Ratsuche erwarten. Bei diesen drei Rahmen wäre es kontraproduktiv, jemand in ein Rätselspiel zu verwickeln, sinnlos, die Selbstdarstellung $\mathrm{zu}$ verzögern und von untergeordneter Bedeutung, sich um Originalität zu bemühen.

Diese Phänomene können hingegen mit dem Spielrahmen plausibel erklärt werden hier ist der Erkenntnisgewinn unserer Studie zu verorten. Das Spielmodell kann erklären, warum Chatkommunikation manchmal belanglos erscheint, warum ernsthafte Probleme nur selten besprochen werden und warum sich an harmlosen Täuschungen kaum jemand stört - das Spiel funktioniert auf diese Weise und verhilft den Spielern zu einem wichtigen Metaziel: dem Spielvergnügen.

\section{Literatur}

Bahl, Anke (1997): Zwischen On- und Offline. Identität und Selbstdarstellung im Internet. München.

Bilandzic, Helena / Koschel, Friederike / Scheufele, Bertram (2001): Theoretisch-heuristische Segmentierung im Prozess der empiriegeleiteten Kategorienbildung. In: Wirth, Werner / Lauf, Edmund (Hrsg.): Inhaltsanalyse: Perspektiven, Probleme, Potenziale. Berlin: $98-116$.

Bilandzic, Helena / Trapp, Bettina (2000): Die Methode des lauten Denkens: Grundlagen des Verfahrens und die Anwendung bei der Untersuchung selektiver Fernsehnutzung bei Jugendlichen. In: Paus-Haase, Ingrid / Schorb, Bernd (Hrsg.): Qualitative Kinder- und Jugendmedienforschung. Theorie und Methoden: ein Arbeitsbuch. München: 183 - 209.

Blanchard, Kendall / Cheska, Alice T. (1985): The anthropology of sport: An introduction. Massachusetts.

Blumer, Herbert (1954): What is wrong with social theory? In: American Sociological Review, 19/1954: 3 - 10.

Crook, Stephan / Taylor, Laurie (1980): Goffman's Version of Reality. In: Ditton, Jason (Hrsg.): The View from Goffman. New York: $233-251$.

Döring, Nicola (1996): Führen Computernetze in die Vereinsamung? In: Gruppendynamik 3/27/1996: $289-307$.

Döring, Nicola (1997a): Identitäten, Beziehungen und Gemeinschaften im Internet. In: Batinic, Bernad (Hrsg.): Internet für Psychologen. Göttingen u. a.: 299 - 336.

Döring, Nicola (1997b): Kommunikation im Internet: Neun theoretische Ansätze. In: Batinic, Bernad (Hrsg.): Internet für Psychologen. Göttingen u. a.: 267 - 298.

Döring, Nicola (1999): Sozialpsychologie des Internet. Göttingen u. a.

Ericsson, Anders K. / Simon, Herbert A. (1993): Protocol Analysis: Verbal Reports As Data. Cambridge/London.

Esser, Hartmut (1999): Soziologie. Spezielle Grundlagen. Band 1: Situationslogik und Handeln. Frankfurt/Main.

Gallery, Heike (2000): »bin ich - klick ich“ - Variable Anonymität im Chat. In: Thimm, Caja (Hrsg.): Soziales im Netz. Sprache, Beziehungen und Kommunikationskulturen im Internet. Opladen/Wiesbaden: $71-88$.

Gelder, Lindsay van (1991): The Strange Case of the Electronic Lover. In: Dunlop, Charles / Kling, Rob (Hrsg.): Computerization and Controversy. New York u. a.: $364-375$.

Goffman, Erving (1977): Rahmen-Analyse. Ein Versuch über die Organisation von Alltagserfahrungen. Frankfurt am Main.

Goffman, Erving (1969): Wir alle spielen Theater. Frankfurt am Main.

Günther, Susanne (1996): Zwischen Scherz und Schmerz - Frotzelaktivitäten in Alltagsinteraktionen. In: Kotthoff, Helga (Hrsg.): Scherzkommunikation. Opladen: 81 - 109.

Hartung, Martin (1996): Ironische Äußerungen in privater Scherzkommunikation. In: Kotthoff, Helga (Hrsg.): Scherzkommunikation. Opladen: $109-143$. 
Henne, Helmut / Rehbock, Helmut (1995): Einführung in die Gesprächsanalyse. 3. Aufl. Berlin u.a.

Hettlage, Robert (1991): Rahmenanalyse - oder die innere Organisation unseres Wissens um die Ordnung der Wirklichkeit. In: Hettlage, Robert/Lenz, Karl (Hrsg.): Erving Goffman - ein soziologischer Klassiker der zweiten Generation. Stuttgart: 95 - 154.

Höflich, Joachim R. (1995): Vom dispersen Publikum zu „elektronischen Gemeinschaften“. Plädoyer für einen kommunikationswissenschaftlichen Blickwinkel. In: Rundfunk und Fernsehen 4/43/1995: $518-537$.

Höflich, Joachim R. (1998): Computerrahmen und Kommunikation. In: Prommer, Elizabeth / Vowe, Gerhard (Hrsg.): Computervermittelte Kommunikation. Öffentlichkeit im Wandel. Konstanz: $141-174$.

Höflich, Joachim R. (1999): „Sex, Lügen und das Internet“. Identität und Glaubwürdigkeit in computervermittelten Beziehungen. In: Rössler, Patrick / Wirth, Werner (Hrsg.): Glaubwürdigkeit im Internet. Feststellungen, Modelle, empirische Befunde. München: 141 - 156.

Höflich, Joachim R. / Gebhardt, Julian (2001): Der Computer als Kontakt- und Beziehungsmedium. In: Medien \& Kommunikationswissenschaft, 1/2001: $24-43$.

Jakobs, Eva-Maria (1998): Mediale Wechsel und Sprache. Entwicklungsstadien elektronischer Schreibwerkzeuge und ihr Einfluß auf Kommunikationsformen. In: Holly, Werner / Biere, Bernd Ulrich (Hrsg.): Medien im Wandel. Opladen: 187 - 209.

Kelle, Uwe (1997): Empirisch begründete Theoriebildung. Zur Logik und Methodologie interpretativer Sozialforschung. 2. Auflage. Weinheim.

Keppler, Angela (1994): Tischgespräche. Über Formen kommunikativer Vergemeinschaftung am Beispiel der Konversation in Familien. Frankfurt am Main.

Kiesler, Sara / Siegel, Jane / McGuire, Timothy (1984): Social Psychological Aspects of ComputerMediated Communication. In: American Psychologist 10/19/1984: 1123 - 1134.

Klemm, Michael / Graner, Lutz (2000): Chatten vor dem Bildschirm: Nutzerkommunikation als Fenster zur alltäglichen Computerkultur. In: Thimm, Caja (Hrsg.): Soziales im Netz. Sprache, Beziehungen und Kommunikationskulturen im Internet. Opladen/Wiesbaden: 156 179.

Krotz, Friedrich (1999): Anonymität als Chance und Glaubwürdigkeit als Problem. Überlegungen zu einigen elementaren Eigenschaften von Kommunikation unter den Bedingungen und Möglichkeiten im Internet. In: Rössler, Patrick / Wirth, Werner (Hrsg.): Glaubwürdigkeit im Internet. Feststellungen, Modelle, empirische Befunde. München: 125 - 140.

Linder, Marc-Olivier / Roos, Johan / Victor, Bart (2001): Play in organizations. Paper presented at EGOS conference July 5-7 2001, Lyon, France.

Mayring, Philipp (1997): Qualitative Inhaltsanalyse. Grundlagen und Techniken. Weinheim.

Mettler-Meibom, Barbara (1990): Wie kommt es zur Zerstörung zwischenmenschlicher Kommunikation? In: Rammert, Werner (Hrsg.): Computerwelten - Alltagswelten. Wie verändert der Computer die soziale Wirklichkeit? Opladen: $65-88$.

Pellegrini, Anthony D. (Ed.) (1995): The future of play theory: A multidisciplinary inquiry into the contributions of Brian Sutton-Smith. Albany, NY.

Rieber, Lloyd P. (1996): Seriously considering play: Designing interactive learning environments based on the blending of microworlds, simulations, and games. Educational Technology Research \& Development, 44(2), 43 - 58

Scherer, Helmut / Wirth, Werner (2002): Ich chatte - wer bin ich? Identität und Selbstdarstellung in virtuellen Kommunikationssituationen. In: Medien \& Kommunikationswissenschaft, 3/2002: $337-358$.

Schmidt, Axel (2000): Chatten. Spiel ohne Grenzen - Spiel mit Grenzen? In: medien praktisch 3/2000: $17-22$.

Stephenson, William (1964): The Ludenic Theory of Newsreading. In: Journalism Quarterly 41: $367-374$.

Stone, Allucquere (1995): The War of Desire and Technology at the Close of the Mechanical Age. Cambridge.

Sutton-Smith, Brian (1997): The Ambiguity of Play. Cambridge.

Turkle, Sherry (1999): Leben im Netz. Identität in Zeiten des Internet. Hamburg. 
Walther, Joseph B. (1996): Computer-Mediated Communication: Impersonal, Interpersonal, and Hyperpersonal Interaction. In: Communication Research 1/23/1996: 3 - 43.

Weinreich, Frank (1997): Moderne Agoren. Nutzungsweisen und Perspektiven von Mailboxsystemen. Wiesbaden.

Willems, Herbert (1997): Rahmen und Habitus. Zum theoretischen und methodischen Ansatz Erving Goffmans: Vergleiche, Anschlüsse und Anwendungen. Frankfurt am Main. 